\title{
On a Conjecture of Ashbaugh and Benguria about Lower Eigenvalues of the Neumann Laplacian
}

\author{
Qiaoling Wang ${ }^{a}$, Changyu Xia ${ }^{a}$ \\ a. Departamento de Matemática, Universidade de Brasilia, 70910-900-Brasilia-DF, Brazil \\ Email: wang@mat.unb.br(Q. Wang),xia@mat.unb.br(C.Xia).
}

\begin{abstract}
In this paper, we prove an isoperimetric inequality for lower order eigenvalues of the free membrane problem on bounded domains in a Euclidean space or a hyperbolic space which strengthens the well-known Szegö-Weinberger inequality and supports a celebrated conjecture of AshbaughBenguria.
\end{abstract}

\section{Introduction}

Let $(M, g)$ be complete Riemannian manifold of dimension $n, n \geq 2$. We denote by $\Delta$ the Laplace operator on $M$. For bounded domain $\Omega$ with smooth boundary in $M$ we consider the free membrane problem

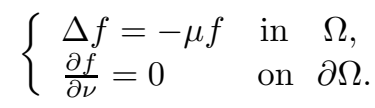

Here $\frac{\partial}{\partial \nu}$ denotes the outward unit normal derivative on $\partial \Omega$. It is well known that the problem (1.1) has discrete spectrum consisting in a sequence

$$
\mu_{0}=0<\mu_{1} \leq \mu_{2} \leq \cdots \rightarrow+\infty .
$$

In the two dimensional case, G. Szego [1] proved via conformal mapping techniques that if $\Omega \subset \mathbb{R}^{2}$ is simply connected, then

$$
\mu_{1}(\Omega) A(\Omega) \leq\left.\left(\mu_{1} A\right)\right|_{d i s k}=\pi p_{1,1}^{2}
$$

where $A$ denotes the area. Later, using more general methods, Weinberger 12 . showed that (1.2) and its $n$-dimensional analogue,

$$
\mu_{1}(\Omega) \leq\left(\frac{\omega_{n}}{|\Omega|}\right)^{2 / n} p_{n / 2,1}^{2},
$$

MSC 2010: 35P15; 49Gxx, 35J05, 33A40.

Key Words: Isoperimetric Inequality, Eigenvalues, Free Membrane Problem, SzegöWeinberger Inequality, Ashbaugh-Benguria Conjecture. 
hold for arbitrary domains in $\mathbb{R}^{2}$ and $\mathbb{R}^{n}$, respectively. Here $J_{v}$ is the Bessel function of the first kind of order $v, p_{v, k}$ is the $k$ th positive zero of the derivative of $x^{l-v} J_{v}(x)$ and $|\Omega|$ denotes the volume of $\Omega$. Szegö and Weinberger also noticed that Szegö's proof of (1.2) for simply connected domains in $\mathbb{R}^{2}$ extends to prove the bound

$$
\frac{1}{\mu_{1}}+\frac{1}{\mu_{2}} \geq \frac{2 A}{\pi p_{1,1}^{2}}
$$

for such domains. The bounds of Szegö and Weinberger are isoperimetric with equality if and only if $\Omega$ is a disk ( $n$-dimensional ball in the case of Weinberger's result (1.9)). A quantitative improvement of (1.2) was made by Brasco and Pratelli in [5] who showed that for any bounded domain with smooth boundary $\Omega \subset \mathbb{R}^{n}$ we have

$$
\omega_{n}^{2 / n} p_{n / 2,1}^{2}-\mu_{1}(\Omega)|\Omega|^{2 / n} \geq c(n) \mathcal{A}(\Omega)^{2} .
$$

Here, $c(n)$ is positive constant depending only on $n$ and $\mathcal{A}(\Omega)$ is the so called Fraenkel asymmetry, defined by

$$
\mathcal{A}(\Omega)=\inf \left\{\frac{|\Omega \Delta B|}{|\Omega|}: B \text { ball in } \mathbb{R}^{\mathrm{n}} \text { such that }|B|=|\Omega|\right\} .
$$

Nadirashvilli obtained in [9] a quantitative improvement of (1.4) which states that there exists a constant $C>0$ such that for every $\Omega \subset \mathbb{R}^{2}$ smooth simply connected bounded open set it holds

$$
\frac{1}{|\Omega|}\left(\frac{1}{\mu_{1}(\Omega)}+\frac{1}{\mu_{2}(\Omega)}\right)-\frac{1}{|B|}\left(\frac{1}{\mu_{1}(B)}+\frac{1}{\mu_{2}(B)}\right) \geq \frac{1}{C} \mathcal{A}(\Omega)^{2},
$$

where $B$ is any disk in $\mathbb{R}^{2}$. On the other hand, Ashbaugh and Benguria [2] showed that

$$
\frac{1}{\mu_{1}(\Omega)}+\cdots+\cdots \frac{1}{\mu_{n}(\Omega)} \geq \frac{n}{n+2}\left(\frac{|\Omega|}{\omega_{n}}\right)^{2 / n}
$$

holds for any $\Omega \subset \mathbb{R}^{n}$. Some generalizations to (1.7) haven been obtained e.g., in 8, [14.

In [2, Ashbaugh and Benguria also proposed the following important

Conjecture I ([2]). For any bounded domain $\Omega$ with smooth boundary in $\mathbb{R}^{n}$, we have

$$
\frac{1}{\mu_{1}(\Omega)}+\frac{1}{\mu_{2}(\Omega)}+\cdots \frac{1}{\mu_{n}(\Omega)} \geq \frac{n\left(|\Omega| / \omega_{n}\right)^{2 / n}}{p_{n / 2,1}^{2}}
$$

with equality holding if and only if $\Omega$ is a ball in $\mathbb{R}^{n}$.

Ashbaugh [1] and Henrot [7] mentioned this conjecture again. 
A more general conjecture might be true. That is, for any bounded domain domain $\Omega$ with smooth boundary in $\mathbb{R}^{n}$, it would hold

$$
\mu_{n}(\Omega) \leq\left(\frac{\omega_{n}}{|\Omega|}\right)^{2 / n} p_{n / 2,1}^{2},
$$

with equality holding if and only if $\Omega$ is a ball in $\mathbb{R}^{n}$.

The Szegö-Weinberger inequality (1.9) has been generalized to bounded domains in a hyperbolic space by Ashbaugh-Benguria [3] and $\mathrm{Xu}$ [14 independently. In his book, Chavel [6] mentioned that one can use Weinberger's method to prove this result. In [3], Ashbaugh-Benguria also proved the SzegöWeinberger inequality for bounded domains in a hemisphere. One can also consider similar estimates for lower order eigenvalues of the Neumann Laplacian for bounded domains in a hyperbolic space or a hemisphere.

Conjecture II. Let $M$ be an n-dimensional complete simply connected Riemannian manifold of constant sectional curvature $\kappa \in\{-1,1\}$ and $\Omega$ be a bounded domain in $M$ which is contained in a hemisphere in the case that $\kappa=1$. Let $B_{\Omega}$ be a geodesic ball in $M$ such that $|\Omega|=\left|B_{\Omega}\right|$ and denote by $\mu_{1}\left(B_{\Omega}\right)$ the first nonzero eigenvalue of the Neumann Laplacian of $B_{\Omega}$. Then the first $n$ non-zero eigenvalues of the Neumann Laplacian of $\Omega$ satisfy

$$
\frac{1}{\mu_{1}(\Omega)}+\frac{1}{\mu_{2}(\Omega)}+\cdots \frac{1}{\mu_{n}(\Omega)} \geq \frac{n}{\mu_{1}\left(B_{\Omega}\right)}
$$

with equality holding if and only if $\Omega$ is isometric to $B_{\Omega}$.

In this paper, we prove an isoperimetric inequality for the sums of the reciprocals of the first $(n-1)$ non-zero eigenvalues of the Neumann Laplacian on bounded domains in $\mathbb{R}^{n}$ or a hyperbolic space which supports the above conjectures.

Theorem 1.1 Let $\Omega$ be a bounded domain with smooth boundary in $\mathbb{R}^{n}$. Then

$$
\frac{1}{\mu_{1}(\Omega)}+\cdots+\frac{1}{\mu_{n-1}(\Omega)} \geq \frac{(n-1)\left(|\Omega| / \omega_{n}\right)^{2 / n}}{p_{n / 2,1}^{2}}
$$

with equality holding if and only if $\Omega$ is a ball in $\mathbb{R}^{n}$.

Theorem 1.2 Let $\mathbb{H}^{n}$ be an n-dimensional hyperbolic space of curvature -1 and $\Omega$ be a bounded domain in $\mathbb{H}^{n}$. Let $B_{\Omega}$ be a geodesic ball in $\mathbb{H}^{n}$ such that $|\Omega|=\left|B_{\Omega}\right|$. Then we have

$$
\frac{1}{\mu_{1}(\Omega)}+\cdots+\frac{1}{\mu_{n-1}(\Omega)} \geq \frac{n-1}{\mu_{1}\left(B_{\Omega}\right)}
$$

with equality holding if and only if $\Omega$ is isometric to $B_{\Omega}$. 


\section{A proof of Theorem 1.1.}

In this section, we shall prove the following result which implies Theorem 1.1.

Theorem 2.1 Let $\Omega$ be a bounded domain with smooth boundary in $\mathbb{R}^{n}$. There exists a positive constant $d(n)$ depending only on $n$ such that the first $(n-1)$ nonzero Neumann eigenvalues of the Laplacian of $\Omega$ satisfy the inequality

$$
\omega_{n}^{2 / n} p_{n / 2,1}^{2}-\frac{(n-1)|\Omega|^{2 / n}}{\frac{1}{\mu_{1}}+\cdots+\frac{1}{\mu_{n-1}}} \geq d(n) \mathcal{A}(\Omega)^{2},
$$

with equality holding if and only if $\Omega$ is an $n$-ball.

Remark. One can easily see that (2.1) strengthens (1.5).

Before proving Theorem 2.1, we recall some known facts we need (Cf. [6], [7], [10]).

Let $\left\{u_{j}\right\}_{j=0}^{\infty}$ be an orthonormal set of eigenfunctions of the problem (1.1), that is,

$$
\left\{\begin{array}{l}
\Delta u_{i}=-\mu_{i} u_{i} \quad \text { in } \quad \Omega \\
\left.\frac{\partial u_{i}}{\partial \nu}\right|_{\partial \Omega}=0 \\
\int_{\Omega} u_{i} u_{j} d v_{g}=\delta_{i j} .
\end{array}\right.
$$

where $d v_{g}$ denotes the volume element of the metric $g$. For each $i=1,2, \cdots$, the variational characterization of $\mu_{i}(\Omega)$ is given by

$$
\mu_{i}(\Omega)=\inf _{u \in H^{1}(\Omega) \backslash\{0\}}\left\{\frac{\int_{\Omega}|\nabla u|^{2} d v_{g}}{\int_{\Omega} u^{2} d v_{g}}: \int_{\Omega} u u_{j} d v_{g}=0, j=0, \cdots, i-1\right\} .
$$

Let $B_{r}$ be a ball of radius $r$ centered at the origin in $\mathbb{R}^{n}$. It is known that $\mu_{1}\left(B_{r}\right)$ has multiplicity $n$, that is, $\mu_{1}\left(B_{r}\right)=\cdots=\mu_{n}\left(B_{r}\right)$. This value can be explicitly computed together with its corresponding eigenfunctions. A basis for the eigenspace corresponding to $\mu_{1}\left(B_{r}\right)$ consists of

$$
\xi_{i}(x)=|x|^{1-\frac{n}{2}} J_{n / 2}\left(\frac{p_{n / 2,1}|x|}{r}\right) \frac{x_{i}}{|x|}, \quad i=1, \cdots, n .
$$

The radial part of $\xi_{i}$

$$
g(|x|)=|x|^{1-\frac{n}{2}} J_{n / 2}\left(\frac{p_{n / 2,1}|x|}{r}\right),
$$

satisfies the differential equation of Bessel type

$$
\left\{\begin{array}{l}
g^{\prime \prime}(t)+\frac{n-1}{t} g^{\prime}(t)+\left(\mu_{1}\left(B_{r}\right)-\frac{n-1}{t^{2}}\right) g(t)=0 \\
g(0)=0, \quad g^{\prime}(r)=0
\end{array}\right.
$$

We can compute

$$
\begin{aligned}
\mu_{1}\left(B_{r}\right) & =\frac{\int_{B_{r}}\left(g^{\prime}(|x|)^{2}+(n-1) \frac{g(|x|)^{2}}{|x|^{2}}\right) d x}{\int_{B_{r}} g(|x|)^{2} d x} \\
& =\left(\frac{p_{n / 2,1}}{r}\right)^{2} .
\end{aligned}
$$


Proof of Theorem [2.1, Let

$$
r=\left(\frac{|\Omega|}{\omega_{n}}\right)^{1 / n}
$$

and define $G:[0,+\infty) \rightarrow \mathbb{R}$ by

$$
G(t)=\left\{\begin{array}{l}
g(t), t \leq r \\
g(r), t>r
\end{array}\right.
$$

We need to choose suitable trial functions $\phi_{i}$ for each of the eigenfunctions $u_{i}$ and insure that these are orthogonal to the preceding eigenfunctions $u_{0}, \cdots, u_{i-1}$. For the $n$ trial functions $\phi_{1}, \phi_{2}, \cdots, \phi_{n}$, we choose:

$$
\phi_{i}=G(|x|) \frac{x_{i}}{|x|}, \text { for } i=1, \cdots, n,
$$

but before we can use these we need to make adjustments so that

$$
\phi_{i} \perp \operatorname{span}\left\{u_{0}, \cdots, u_{i-1}\right\}
$$

in $L^{2}(\Omega)$. In order to do this, let us fix an orthonormal basis $\left\{e_{i}\right\}_{i=1}^{n}$ of $\mathbb{R}^{n}$. From the well-know arguments of Weinberger in [12] by using the Brouwer fixed point theorem, we know that it is always possible to choose the origin of $\mathbb{R}^{n}$ so that

$$
\int_{\Omega}\left\langle x, e_{i}\right\rangle \frac{G(|x|)}{|x|} d x=0, \quad i=1, \cdots, n,
$$

that is, $\left\langle x, e_{i}\right\rangle \frac{G(|x|)}{|x|} \perp u_{0}$ (which is actually just the constant function $1 / \sqrt{|\Omega|}$ ). Here $d x$ and $\langle$,$\rangle denote the standard Lebesgue measure and the inner product$ of $\mathbb{R}^{n}$, respectively. Now we show that there exists a new orthonormal basis $\left\{e_{i}^{\prime}\right\}_{i=1}^{n}$ of $\mathbb{R}^{n}$ such that

$$
\left\langle x, e_{i}^{\prime}\right\rangle \frac{G(|x|)}{|x|} \perp u_{j},
$$

for $j=1, \cdots, i-1$ and $i=2, \cdots, n$. To see this, we define an $n \times n$ matrix $Q=\left(q_{i j}\right)$ by

$$
q_{i j}=\int_{\Omega}\left\langle x, e_{i}\right\rangle \frac{G(|x|)}{|x|} u_{j}(x) d x, i, j=1,2, \cdots, n .
$$

Using the orthogonalization of Gram and Schmidt (QR-factorization theorem), we know that there exist an upper triangle matrix $T=\left(T_{i j}\right)$ and an orthogonal matrix $U=\left(a_{i j}\right)$ such that $T=U Q$, i.e.,

$$
T_{i j}=\sum_{k=1}^{n} a_{i k} q_{k j}=\int_{\Omega} \sum_{k=1}^{n} a_{i k}\left\langle x, e_{k}\right\rangle \frac{G(|x|)}{|x|} u_{j}(x) d x=0, \quad 1 \leq j<i \leq n .
$$


Letting $e_{i}^{\prime}=\sum_{k=1}^{n} a_{i k} e_{k}, i=1, \ldots, n$; we arrive at (2.13). Let us denote by $x_{1}, x_{2}, \cdots, x_{n}$ the coordinate functions with respect to the base $\left\{e_{i}^{\prime}\right\}_{i=1}^{n}$, that is, $x_{i}=\left\langle x, e_{i}^{\prime}\right\rangle, x \in \mathbb{R}^{n}$. From (2.12) and (2.13), we have

$$
\int_{\Omega} \phi_{i} u_{j} d x=\int_{\Omega} G(|x|) \frac{x_{i}}{|x|} u_{j}(x) d x=0, i=1, \cdots, n, j=0, \cdots, i-1 .
$$

It then follows from the variational characterization (2.3) that

$$
\mu_{i} \int_{\Omega} \phi_{i}^{2} d x \leq \int_{\Omega}\left|\nabla \phi_{i}\right|^{2} d x, i=1, \cdots, n .
$$

Substituting

$$
\begin{aligned}
\left|\nabla \phi_{i}\right|^{2} & =G^{\prime}(|x|)^{2} \frac{x_{i}^{2}}{|x|^{2}}+\frac{G(|x|)^{2}}{|x|^{2}}\left(1-\frac{x_{i}^{2}}{|x|^{2}}\right) \\
& =\frac{G(|x|)^{2}}{|x|^{2}}+\left(G^{\prime}(|x|)^{2}-\frac{G(|x|)^{2}}{|x|^{2}}\right) \frac{x_{i}^{2}}{|x|^{2}}
\end{aligned}
$$

into (2.16) and dividing by $\mu_{i}$, one gets for $i=1, \cdots, n$ that

$$
\int_{\Omega} \phi_{i}^{2} d x \leq \frac{1}{\mu_{i}} \int_{\Omega} \frac{G(|x|)^{2}}{|x|^{2}} d x+\frac{1}{\mu_{i}} \int_{\Omega}\left(G^{\prime}(|x|)^{2}-\frac{G(|x|)^{2}}{|x|^{2}}\right) \frac{x_{i}^{2}}{|x|^{2}} d x .
$$

Summing over $i$, we get

$$
\begin{aligned}
\int_{\Omega} G(|x|)^{2} d x \leq & \sum_{i=1}^{n} \frac{1}{\mu_{i}} \int_{\Omega} \frac{G(|x|)^{2}}{|x|^{2}} d x \\
& +\sum_{i=1}^{n} \frac{1}{\mu_{i}} \int_{\Omega}\left(G^{\prime}(|x|)^{2}-\frac{G(|x|)^{2}}{|x|^{2}}\right) \frac{x_{i}^{2}}{|x|^{2}} d x .
\end{aligned}
$$

Since

$$
\begin{aligned}
\sum_{i=1}^{n} \frac{1}{\mu_{i}} \frac{x_{i}^{2}}{|x|^{2}} & =\sum_{i=1}^{n-1} \frac{1}{\mu_{i}} \frac{x_{i}^{2}}{|x|^{2}}+\frac{1}{\mu_{n}} \frac{x_{n}^{2}}{|x|^{2}} \\
& =\sum_{i=1}^{n-1} \frac{1}{\mu_{i}} \frac{x_{i}^{2}}{|x|^{2}}+\frac{1}{\mu_{n}}\left(1-\sum_{i=1}^{n-1} \frac{x_{i}^{2}}{|x|^{2}}\right)
\end{aligned}
$$


we have

$$
\begin{aligned}
& \sum_{i=1}^{n} \frac{1}{\mu_{i}} \int_{\Omega}\left(G^{\prime}(|x|)^{2}-\frac{G(|x|)^{2}}{|x|^{2}}\right) \frac{x_{i}^{2}}{|x|^{2}} d x \\
= & \sum_{i=1}^{n-1} \frac{1}{\mu_{i}} \int_{\Omega}\left(G^{\prime}(|x|)^{2}-\frac{G(|x|)^{2}}{|x|^{2}}\right) \frac{x_{i}^{2}}{|x|^{2}} d x \\
& +\frac{1}{\mu_{n}} \int_{\Omega}\left(G^{\prime}(|x|)^{2}-\frac{G(|x|)^{2}}{|x|^{2}}\right) d x \\
& -\frac{1}{\mu_{n}} \int_{\Omega}\left(G^{\prime}(|x|)^{2}-\frac{G(|x|)^{2}}{|x|^{2}}\right) \sum_{i=1}^{n-1} \frac{x_{i}^{2}}{|x|^{2}} d x \\
= & \sum_{i=1}^{n-1} \int_{\Omega}\left(\frac{1}{\mu_{i}}-\frac{1}{\mu_{n}}\right)\left(G^{\prime}(|x|)^{2}-\frac{G(|x|)^{2}}{|x|^{2}}\right) \frac{x_{i}^{2}}{|x|^{2}} d x \\
& +\frac{1}{\mu_{n}} \int_{\Omega}\left(G^{\prime}(|x|)^{2}-\frac{G(|x|)^{2}}{|x|^{2}}\right) d x .
\end{aligned}
$$

Lemma 2.2 We have $\left.g^{\prime}\right|_{[0, r)}>0,\left.g\right|_{(0, r]}>0$ and $g^{\prime}(t)-\frac{g(t)}{t} \leq 0, \forall t \in(0, r]$.

Proof of Lemma 2.2. The Bessel function of the first kind $J_{v}(t)$ is given by

$$
J_{v}(t)=\sum_{k=0}^{+\infty} \frac{(-1)^{k}\left(\frac{t}{2}\right)^{2 k+v}}{k ! \Gamma(k+v+1)},
$$

which, combining with (2.5), gives

$$
g(t)=\left(\frac{p_{n / 2,1}}{2 r}\right)^{\frac{n}{2}} t \sum_{k=0}^{+\infty} \frac{(-1)^{k}\left(\frac{p_{n / 2,1}}{2 r} t\right)^{2 k}}{k ! \Gamma\left(k+\frac{n}{2}+1\right)} .
$$

Thus, $g(0)=0, g^{\prime}(0)>0$. Since $r$ is the first positive zero of $g^{\prime}$, we have $\left.g\right|_{(0, r]}>0$ and $\left.g^{\prime}\right|_{[0, r)}>0$. Observe that

$$
\lim _{t \rightarrow 0}\left(g^{\prime}(t)-\frac{g(t)}{t}\right)=0, \quad g^{\prime}(r)-\frac{g(r)}{r}<0 .
$$

Let us assume by contradiction that there exists a $t_{0} \in(0, r)$ such that

$$
g^{\prime}\left(t_{0}\right)-\frac{g\left(t_{0}\right)}{t_{0}}>0
$$

In this case, we know from (2.24) that the function $g^{\prime}(t)-\frac{g(t)}{t}$ attains its maximum at some $t_{1} \in(0, r)$ and so we have

$$
g^{\prime \prime}\left(t_{1}\right)-\frac{t_{1} g^{\prime}\left(t_{1}\right)-g\left(t_{1}\right)}{t_{1}^{2}}=0 .
$$


From (2.6), we have

$$
g^{\prime \prime}\left(t_{1}\right)+\frac{n-1}{t_{1}} g^{\prime}\left(t_{1}\right)+\left(\mu_{1}\left(B_{r}\right)-\frac{n-1}{t_{1}^{2}}\right) g\left(t_{1}\right)=0 .
$$

Eliminating $g^{\prime \prime}\left(t_{1}\right)$ from (2.26) and (2.27), we get

$$
\frac{n}{t_{1}}\left(g^{\prime}\left(t_{1}\right)-\frac{g\left(t_{1}\right)}{t_{1}}\right)=-\mu_{1}\left(B_{r}\right) g\left(t_{1}\right)<0 .
$$

This is a contradiction and completes the proof of Lemma 2.1.

From Lemma 2.1 and the definition of $G$, we know that

$$
G^{\prime}(|x|)^{2}-\frac{G(|x|)^{2}}{|x|^{2}} \leq 0 \quad \text { on } \Omega .
$$

Hence

$$
\sum_{i=1}^{n-1} \int_{\Omega}\left(\frac{1}{\mu_{i}}-\frac{1}{\mu_{n}}\right)\left(G^{\prime}(|x|)^{2}-\frac{G(|x|)^{2}}{|x|^{2}}\right) \frac{x_{i}^{2}}{|x|^{2}} d x \leq 0 .
$$

Combining (2.19), (2.21) and (2.30), one gets

$$
\begin{aligned}
\int_{\Omega} G(|x|)^{2} d x \leq & \frac{1}{\mu_{n}} \int_{\Omega}\left(G^{\prime}(|x|)^{2}-\frac{G(|x|)^{2}}{|x|^{2}}\right) d x \\
& +\sum_{i=1}^{n} \frac{1}{\mu_{i}} \int_{\Omega} \frac{G(|x|)^{2}}{|x|^{2}} d x \\
= & \frac{1}{\mu_{n}} \int_{\Omega} G^{\prime}(|x|)^{2}+\sum_{i=1}^{n-1} \frac{1}{\mu_{i}} \int_{\Omega} \frac{G(|x|)^{2}}{|x|^{2}} d x \\
\leq & \frac{1}{n-1} \sum_{i=1}^{n-1} \frac{1}{\mu_{i}} \int_{\Omega}\left(G^{\prime}(|x|)^{2}+(n-1) \frac{G(|x|)^{2}}{|x|^{2}}\right) d x
\end{aligned}
$$

that is,

$$
\frac{n-1}{\sum_{i=1}^{n-1} \frac{1}{\mu_{i}}} \int_{\Omega} G(|x|)^{2} d x \leq \int_{\Omega}\left(G^{\prime}(|x|)^{2}+(n-1) \frac{G(|x|)^{2}}{|x|^{2}}\right) d x .
$$

Using the fact that $G(t)$ is increasing, one gets

$$
\begin{aligned}
\int_{\Omega} G(|x|)^{2} d x & =\int_{\Omega \cap B_{r}} G(|x|)^{2} d x+\int_{\Omega \backslash B_{r}} G(|x|)^{2} d x \\
& \geq \int_{\Omega \cap B_{r}} G(|x|)^{2} d x+g(r)^{2}\left|\Omega \backslash B_{r}\right| \\
& =\int_{\Omega \cap B_{r}} g(|x|)^{2} d x+g(r)^{2}\left|B_{r} \backslash \Omega\right| \\
& \geq \int_{\Omega_{n} B_{r}} g(|x|)^{2}+\int_{B_{r} \backslash \Omega} g(|x|)^{2} d x \\
& =\int_{B_{r}} g(|x|)^{2} d x
\end{aligned}
$$


which, combining with (2.32), gives

$$
\frac{n-1}{\sum_{i=1}^{n-1} \frac{1}{\mu_{i}}} \int_{B_{r}} g(|x|)^{2} d x \leq \int_{\Omega}\left(G^{\prime}(|x|)^{2}+(n-1) \frac{G(|x|)^{2}}{|x|^{2}}\right) d x .
$$

We know from (2.7) that

$$
\begin{aligned}
\left(\frac{p_{n / 2,1}}{r}\right)^{2} \int_{B_{r}} g(|x|)^{2} d x & =\int_{B_{r}}\left(g^{\prime}(|x|)^{2}+(n-1) \frac{g(|x|)^{2}}{|x|^{2}}\right) d x \\
& =\int_{B_{r}}\left(G^{\prime}(|x|)^{2}+(n-1) \frac{G(|x|)^{2}}{|x|^{2}}\right) d x
\end{aligned}
$$

Consequently, we have

$$
\begin{aligned}
& \left(\left(\frac{p_{n / 2,1}}{r}\right)^{2}-\frac{n-1}{\sum_{i=1}^{n-1} \frac{1}{\mu_{i}}}\right) \int_{B_{r}} g(|x|)^{2} d x \\
\geq & \int_{B_{r}}\left(G^{\prime}(|x|)^{2}+(n-1) \frac{G(|x|)^{2}}{|x|^{2}}\right) d x-\int_{\Omega}\left(G^{\prime}(|x|)^{2}+(n-1) \frac{G(|x|)^{2}}{|x|^{2}}\right) d x .
\end{aligned}
$$

We have

$\frac{d}{d t}\left[G^{\prime}(t)^{2}+(n-1) \frac{G(t)^{2}}{t^{2}}\right]=2 G^{\prime}(t) G^{\prime \prime}(t)+2(n-1)\left(t G(t) G^{\prime}(t)-G(t)^{2}\right) / t^{3}$.

For $t>r$ this is negative since $G$ is constant there. For $t \leq r$ we use the differential equation (2.6) to obtain

$$
\frac{d}{d t}\left[G^{\prime}(t)^{2}+(n-1) \frac{G(t)^{2}}{t^{2}}\right]=-2 \mu_{1}\left(B_{r}\right) G G^{\prime}-(n-1)\left(t G^{\prime}-G\right)^{2} / t^{3}<0 .
$$

Thus the function $G^{\prime}(t)^{2}+(n-1) \frac{G(t)^{2}}{t^{2}}$ is decreasing for $t>0$.

Lemma 2.3 ([5]) Let $f: \mathbb{R}_{+} \rightarrow \mathbb{R}_{+}$be a decreasing function. Then we have

$$
\int_{B_{r}} f(|x|) d x-\int_{\Omega} f(|x|) d x \geq n \omega_{n} \int_{\rho_{1}}^{\rho_{2}}|f(t)-f(r)| t^{n-1} d t .
$$

Here

$$
\rho_{1}=\left(\frac{\left|\Omega \cap B_{r}\right|}{\omega_{n}}\right)^{\frac{1}{n}} \quad \text { and } \quad \rho_{2}=\left(\frac{|\Omega|+\left|\Omega \backslash B_{r}\right|}{\omega_{n}}\right)^{\frac{1}{n}} .
$$

Taking $f(t)=G^{\prime}(t)^{2}+(n-1) \frac{G(t)^{2}}{t^{2}}$ in Lemma 2.3, we obtain

$$
\begin{aligned}
& \int_{B_{r}}\left(G^{\prime}(|x|)^{2}+(n-1) \frac{G(|x|)^{2}}{|x|^{2}}\right) d x-\int_{\Omega}\left(G^{\prime}(|x|)^{2}+(n-1) \frac{G\left(|x|^{2}\right.}{|x|^{2}}\right) d x \\
\geq & n \omega_{n} \int_{r}^{\rho_{2}}|f(t)-f(r)| t^{n-1} d t \\
= & n \omega_{n} \int_{r}^{\rho_{2}}(f(r)-f(t)) t^{n-1} d t
\end{aligned}
$$


Observe that

$$
(f(r)-f(t)) t^{n-1}=(n-1) g(r)^{2}\left(\frac{1}{r^{2}}-\frac{1}{t^{2}}\right) t^{n-1}, \quad \text { for } \rho_{2} \geq t \geq r .
$$

Therefore,

$$
\begin{aligned}
& \int_{r}^{\rho_{2}}(f(r)-f(t)) t^{n-1} d t \\
= & g(r)^{2} \cdot \begin{cases}\frac{n-1}{n r^{2}}\left(\rho_{2}^{n}-r^{n}\right)-\frac{n-1}{n-2}\left(\rho_{2}^{n-2}-r^{n-2}\right), & \text { if } n>2, \\
\frac{1}{2 r^{2}}\left(\rho_{2}^{2}-r^{2}\right)-\ln \frac{\rho_{2}}{r}, & \text { if } n=2 .\end{cases}
\end{aligned}
$$

By using the definition of $\rho_{2}$ we have when $n>2$,

$$
\begin{aligned}
\rho_{2}^{n-2}-r^{n-2} & =r^{n-2}\left[\left(1+\frac{\left|\Omega \backslash B_{r}\right|}{|\Omega|}\right)^{\frac{n-2}{n}}-1\right] \\
& \leq r^{n-2}\left(\frac{n-2}{n} \frac{\left|\Omega \backslash B_{r}\right|}{|\Omega|}-\frac{(n-2) 2^{-\frac{2}{n}-1}}{n^{2}}\left(\frac{\left|\Omega \backslash B_{r}\right|}{|\Omega|}\right)^{2}\right)
\end{aligned}
$$

thanks to the elementary inequality

$$
(1+t)^{\delta} \leq 1+\delta t+\frac{\delta(\delta-1)}{2} \cdot 2^{\delta-2} t^{2}, \forall \delta \in(0,1), \forall t \in[0,1],
$$

and when $n=2$,

$$
\begin{aligned}
\ln \frac{\rho_{2}}{r} & =\frac{1}{2} \ln \left(1+\frac{\left|\Omega \backslash B_{r}\right|}{|\Omega|}\right) \\
& \leq \frac{1}{2}\left(\frac{\left|\Omega \backslash B_{r}\right|}{|\Omega|}-\frac{1}{4}\left(\frac{\left|\Omega \backslash B_{r}\right|}{|\Omega|}\right)^{2}\right),
\end{aligned}
$$

thanks to the elementary inequality

$$
\ln (1+t) \leq t-\frac{t^{2}}{4}, \quad \forall t \in[0,1] .
$$

Since $\left|B_{r}\right|=|\Omega|$, we have $\left|\Omega \Delta B_{r}\right|=2\left|\Omega \backslash B_{r}\right|$ and so

$$
\frac{\left|\Omega \backslash B_{r}\right|}{|\Omega|} \geq \frac{1}{2} \mathcal{A}(\Omega)
$$

It then follows by substituting (2.42) and (2.43) into (2.41) that

$$
\begin{aligned}
& \int_{r}^{\rho_{2}}(f(r)-f(t)) t^{n-1} d t \\
= & g(r)^{2}\left(\frac{\left|\Omega \backslash B_{r}\right|}{|\Omega|}\right)^{2} \cdot \begin{cases}r^{n-2} \cdot \frac{(n-1) 2^{-\frac{2}{n}-1}}{n^{2}}, & \text { if } n>2, \\
\frac{1}{8}, & \text { if } n=2 .\end{cases} \\
\geq & \frac{1}{4} g(r)^{2} \mathcal{A}(\Omega)^{2} \cdot \begin{cases}r^{n-2} \cdot \frac{(n-1) 2^{-\frac{2}{n}-1}}{n^{2}}, & \text { if } n>2, \\
\frac{1}{8}, & \text { if } n=2 .\end{cases}
\end{aligned}
$$


Thus, concerning the right hand side of (2.36), one gets from (2.39) and (2.44) that

$$
\begin{aligned}
& \int_{B_{r}}\left(G^{\prime}(|x|)^{2}+(n-1) \frac{G(|x|)^{2}}{|x|^{2}}\right) d x-\int_{\Omega}\left(G^{\prime}(|x|)^{2}+(n-1) \frac{G\left(|x|^{2}\right.}{|x|^{2}}\right) d x \\
& \geq \frac{\omega_{n}}{4} g(r)^{2} \mathcal{A}(\Omega)^{2} \cdot \begin{cases}r^{n-2} \cdot \frac{(n-1) 2^{-\frac{2}{n}-1}}{n}, & \text { if } n>2, \\
\frac{1}{4}, & \text { if } n=2,\end{cases} \\
& =\frac{\omega_{n}}{4} J_{n / 2}\left(p_{n / 2,1}\right)^{2} \mathcal{A}(\Omega)^{2} \cdot \begin{cases}\frac{(n-1) 2^{-\frac{2}{n}-1}}{n}, & \text { if } n>2, \\
\frac{1}{4}, & \text { if } n=2,\end{cases} \\
& \equiv \alpha(n) \mathcal{A}(\Omega)^{2} \text {. }
\end{aligned}
$$

Concerning the left hand side of (2.36), we have

$$
\begin{aligned}
& \left(\left(\frac{p_{n / 2,1}}{r}\right)^{2}-\frac{n-1}{\sum_{i=1}^{n-1} \frac{1}{\mu_{i}}}\right) \int_{B_{r}} g(|x|)^{2} d x \\
= & \left(\left(\frac{p_{n / 2,1}}{r}\right)^{2}-\frac{n-1}{\sum_{i=1}^{n-1} \frac{1}{\mu_{i}}}\right) r^{2} \int_{\{|y| \leq 1\}}|y|^{2-n} J_{\frac{n}{2}}\left(p_{n / 2,1}|y|\right)^{2} d y \\
= & \left(p_{n / 2,1}^{2} \omega_{n}^{2 / n}-\frac{(n-1)|\Omega|^{2 / n}}{\sum_{i=1}^{n-1} \frac{1}{\mu_{i}}}\right) \beta(n),
\end{aligned}
$$

where

$$
\beta(n)=\omega_{n}^{-2 / n} \int_{\{|y| \leq 1\}}|y|^{2-n} J_{\frac{n}{2}}\left(p_{n / 2,1}|y|\right)^{2} d y .
$$

Combining (2.36), (2.45) and (2.46), we obtain

$$
p_{n / 2,1}^{2} \omega_{n}^{2 / n}-\frac{(n-1)|\Omega|^{2 / n}}{\sum_{i=1}^{n-1} \frac{1}{\mu_{i}}} \geq \alpha(n) \beta(n)^{-1} \mathcal{A}(\Omega)^{2} \equiv d(n) \mathcal{A}(\Omega)^{2} .
$$

Moreover, we can see that equality holds in (2.47) only when $\Omega$ is a ball. This completes the proof of Theorem 2.1.

\section{A Proof of Theorem 1.2}

In this section, we shall prove Theorem 1.2. Firstly, we list some important facts we need. About each point $p \in \mathbb{H}^{n}$ there exists a coordinate system $(t, \xi) \in[0,+\infty) \times \mathbb{S}^{n-1}$ relative to which the Riemannian metric reads as

$$
d s^{2}=d t^{2}+\sinh ^{2} t d \sigma^{2},
$$

where $d \sigma^{2}$ is the canonical metric on the $(n-1)$-dimensional unit sphere $\mathbb{S}^{n-1}$. 
Lemma 3.1 (Cf. [6], [14]). Let $B(p, r)$ be a geodesic ball of radius $r$ with center $p$ in $\mathbb{H}^{n}$. Then the eigenfunction corresponding to the first nonzero eigenvalue $\mu_{1}(B(p, r))$ of the Neumann problem on $B(p, r)$ must be

$$
h(t, \xi)=f(t) \omega(\xi), \xi \in \mathbb{S}^{n-1},
$$

where $\omega(\xi)$ is an eigenfunction corresponding to the first nonzero eigenvalue of $\mathbb{S}^{n-1}, f$ satisfies

$$
\left\{\begin{array}{l}
f^{\prime \prime}+(n-1) \operatorname{coth} t+\left(\mu_{1}(B(p, r))-\frac{n-1}{\sinh ^{2} t}\right) f=0, \\
f(0)=f^{\prime}(r)=0,\left.f^{\prime}\right|_{[0, r)} \neq 0,
\end{array}\right.
$$

and

$$
\mu_{1}(B(p, r))=\frac{\int_{B(p, r)}\left(f^{\prime}(t)^{2}+(n-1) \frac{f(t)^{2}}{\sinh ^{2} t}\right) d v}{\int_{B(p, r)} f(t)^{2} d v} .
$$

Proof of Theorem 1.2. Assume that the radius of $B_{\Omega}$ is $r$. Let $f$ be as in Lemma 3.1. Noticing $f(t) \neq 0$ when $0<t \leq r$, we may assume that $f(t)>0$ for $0<t \leq r$ and so $f$ is nondecreasing on $[0, r]$. Let $\left\{\mathbf{e}_{i}\right\}_{i=1}^{n}$ be an orthonormal basis of $\mathbb{R}^{n}$ and set $\omega_{i}(\xi)=\left\langle\mathbf{e}_{i}, \xi\right\rangle, \xi \in \mathbb{S}^{n-1} \subset \mathbb{R}^{n}$. Define

$$
F(t)=\left\{\begin{array}{l}
f(t), \quad t \leq r \\
f(r), \quad t>r
\end{array}\right.
$$

Let us take a point $p \in \mathbb{H}^{n}$ such that in the above coordinate system at $p$ we have

$$
\int_{\Omega} F(t) \omega_{i}(\xi) d v=0, \quad i=1, \cdots, n .
$$

Here, $d v$ is the volume element of $\mathbb{H}^{n}$. By using the same arguments as in the proof of Theorem 2.1, we can assume further that

$$
\int_{\Omega} F(t) \omega_{i}(\xi) u_{j} d v=0
$$

for $i=2,3, \cdots, n$ and $j=1, \cdots, i-1$. Here $\left\{u_{i}\right\}_{i=0}^{+\infty}$ is a orthonormal set of eigenfunctions corresponding to the eigenvalues $\left\{\mu_{i}(\Omega)\right\}_{i=0}^{+\infty}$. Hence, we conclude from the Rayleigh-Ritz variational characterization (2.3) that

$$
\begin{aligned}
& \mu_{i}(\Omega) \int_{\Omega} F(t)^{2} \omega_{i}^{2}(\xi) d v \\
\leq & \int_{\Omega}\left|\nabla\left(F(t) \omega_{i}(\xi)\right)\right|^{2} d v \\
= & \int_{\Omega}\left(\left|F^{\prime}(t)\right|^{2} \omega_{i}^{2}(\xi)+F^{2}(t)\left|\tilde{\nabla} \omega_{i}(\xi)\right|^{2} \sinh ^{-2} t\right) d v, i=1, \cdots, n,
\end{aligned}
$$


where $\tilde{\nabla}$ denotes the gradient operator of $\mathbb{S}^{n-1}$. Thus

$$
\begin{aligned}
& \int_{\Omega} F(t)^{2} \omega_{i}^{2}(\xi) d v \\
\leq & \frac{1}{\mu_{i}(\Omega)} \int_{\Omega}\left|F^{\prime}(t)\right|^{2} \omega_{i}^{2}(\xi) d v+\frac{1}{\mu_{i}(\Omega)} \int_{\Omega} F^{2}(t)\left|\tilde{\nabla} \omega_{i}(\xi)\right|^{2} \sinh ^{-2} t d v .
\end{aligned}
$$

Observing $F^{\prime}(t)=0, t \geq r$, one gets

$$
\begin{aligned}
\int_{\Omega}\left|F^{\prime}(t)\right|^{2} \omega_{i}^{2}(\xi) d v & =\int_{\Omega \cap B(p, r)}\left|F^{\prime}(t)\right|^{2} \omega_{i}^{2}(\xi) d v \\
& \leq \int_{B(p, r)}\left|F^{\prime}(t)\right|^{2} \omega_{i}^{2}(\xi) d v \\
& =\int_{0}^{r} \int_{\mathbb{S}^{n-1}}\left|F^{\prime}(t)\right|^{2} \omega_{i}^{2}(\xi) \sinh ^{n-1} t d A d t \\
& =\frac{1}{n} \int_{0}^{r} \int_{\mathbb{S}^{n-1}}\left|F^{\prime}(t)\right|^{2} \sinh ^{n-1} t d A d t \\
& =\frac{1}{n} \int_{B(p, r)}\left|F^{\prime}(t)\right|^{2} d v,
\end{aligned}
$$

where $d A$ denotes the area element of $\mathbb{S}^{n-1}$. Since

$$
\left|\tilde{\nabla} \omega_{i}(\xi)\right| \leq 1, \quad \sum_{i=1}^{n}\left|\tilde{\nabla} \omega_{i}(\xi)\right|^{2}=n-1,
$$

we have

$$
\begin{aligned}
& \sum_{i=1}^{n} \frac{1}{\mu_{i}(\Omega)}\left|\tilde{\nabla} \omega_{i}(\xi)\right|^{2} \\
= & \sum_{i=1}^{n-1} \frac{1}{\mu_{i}(\Omega)}\left|\tilde{\nabla} \omega_{i}(\xi)\right|^{2}+\frac{1}{\mu_{n}(\Omega)} \sum_{i=1}^{n-1}\left(1-\left|\tilde{\nabla} \omega_{i}(\xi)\right|^{2}\right) \\
\leq & \sum_{i=1}^{n-1} \frac{1}{\mu_{i}(\Omega)}\left|\tilde{\nabla} \omega_{i}(\xi)\right|^{2}+\sum_{i=1}^{n-1} \frac{1}{\mu_{i}(\Omega)}\left(1-\left|\tilde{\nabla} \omega_{i}(\xi)\right|^{2}\right) \\
= & \sum_{i=1}^{n-1} \frac{1}{\mu_{i}(\Omega)} .
\end{aligned}
$$

Summing on $i$ from 1 to $n$ in (3.9) and using (3.10) and (3.12), we get

$$
\begin{aligned}
& \int_{\Omega} F(t)^{2} d v \\
\leq & \sum_{i=1}^{n} \frac{1}{n \mu_{i}(\Omega)} \int_{B(p, r)}\left|F^{\prime}(t)\right|^{2} d v+\sum_{i=1}^{n-1} \frac{1}{\mu_{i}(\Omega)} \int_{\Omega} F^{2}(t) \sinh ^{-2} t d v .
\end{aligned}
$$

We need the following lemma. 
Lemma 3.2 The function $h(t)=\frac{F(t)}{\sinh t}$ is decreasing.

Proof of Lemma 3.2. Observe that

$$
\lim _{t \rightarrow 0} h(t)=f^{\prime}(0)
$$

Let us show that

$$
\gamma(t) \equiv f^{\prime}(t)-\operatorname{coth} t f(t) \leq 0, t \in(0, r] .
$$

Since

$$
\lim _{t \rightarrow 0} \gamma(t)=0, \gamma(r)=-\operatorname{coth} r f(r)<0,
$$

if $\gamma\left(t_{0}\right)>0$ for some $t_{0} \in(0, r)$, then $\gamma$ attains its maximum at some $t_{1} \in(0, r)$ and so

$$
0=\gamma^{\prime}\left(t_{1}\right)=f^{\prime \prime}\left(t_{1}\right)+\frac{f\left(t_{1}\right)}{\sinh ^{2} t_{1}}-\operatorname{coth} t_{1} f^{\prime}\left(t_{1}\right) .
$$

We have from (3.3) that

$$
f^{\prime \prime}\left(t_{1}\right)+(n-1) \operatorname{coth} t_{1} f^{\prime}\left(t_{1}\right)+\mu_{1}(B(r)) f\left(t_{1}\right)-\frac{n-1}{\sinh ^{2} t_{1}} f\left(t_{1}\right)=0 .
$$

Hence

$$
f^{\prime}\left(t_{1}\right)-\frac{f\left(t_{1}\right)}{\cosh t_{1} \sinh t_{1}}=-\frac{\mu_{1}(B(r)) f\left(t_{1}\right) \sinh t_{1}}{n \cosh t_{1}}<0,
$$

which contradicts to

$$
f^{\prime}\left(t_{1}\right)-\operatorname{coth} t_{1} f\left(t_{1}\right)>0 .
$$

Thus (3.14) holds. Consequently $h^{\prime}(t) \leq 0, \forall t \in(0, r]$ and $h$ is decreasing. The proof of Lemma 3.2 is completed.

Now we go on the proof of Theorem 1.2. Since $F$ is increasing and $\frac{F(t)}{\sinh t}$ is decreasing, we can use the same arguments as in the proof of (2.33) to conclude that

$$
\int_{\Omega} F(t)^{2} d v \geq \int_{B(p, r)} f(t)^{2} d v
$$

and

$$
\int_{\Omega} \frac{F(t)^{2}}{\sinh ^{2} t} d v \leq \int_{B(p, r)} \frac{f(t)^{2}}{\sinh ^{2} t} d v .
$$

Substituting (3.20) and (3.21) into (3.13), one gets

$$
\begin{aligned}
\frac{1}{n-1} \sum_{i=1}^{n-1} \frac{1}{\mu_{i}(\Omega)} & \geq \frac{\int_{B(p, r)} f(t)^{2} d v}{\int_{B(p, r)}\left(f^{\prime}(t)^{2}+(n-1) \frac{f(t)^{2}}{\sinh ^{2} t}\right) d v} \\
& =\frac{1}{\mu_{1}(B(p, r))}
\end{aligned}
$$

and equality holds if and only if $\Omega=B(p, r)$. This completes the proof of Theorem 1.2 , 


\section{Acknowledgments}

Q. Wang was partially supported by CNPq, Brazil (Grant No. 307089/2014-2).

C. Xia was partially supported by CNPq, Brazil (Grant No. 306146/2014-2).

\section{References}

[1] M. S. Ashbaugh, Open problems on eigenvalues of the Laplacian, Analytic and Geometric Inequalities and Applications. (1999), 13-28.

[2] M. S. Ashbaugh and R. D. Benguria, Universal bounds for the low eigenvalues of Neumann Laplacians in $N$ dimensions. Siam J. Math. Anal. 24 (1993), 557-570.

[3] M. S. Ashbaugh and R. D. Benguria, Sharp upper bound to the first nonzero Neumann eigenvalue for bounded domains in spaces of constant curvature, J. London Math. Soc. 52 (1995) 402-416.

[4] L. Brasco and G. De Philippis, Spectral inequalities in quantitative form. Shape optimization and spectral theory, 201-281, De Gruyter Open, Warsaw, 2017.

[5] L. Brasco, A. Pratelli, Sharp stability of some spectral inequalities, Geom. Funct. Anal., 22 (2012), 107-135.

[6] I. Chavel, Eigenvalues in Riemannian geometry (Academic, New York, 1984).

[7] A. Henrot, Extremum problems for eigenvalues of elliptic operators, Birkhäuser Verlag, Basel-Boston-Berlin, x + 202 pp., 2006. ISBN 978-376437705-2.

[8] G. N. Hile, Z. Xu, Inequalities for sums of the reciprocals of eigenvalues, J. Math. Anal. Appl. 180 (1993) 412-430.

[9] N. Nadirashvili, Conformal maps and isoperimetric inequalities for eigenvalues of the Neumann problem. Proceedings of the Ashkelon Workshop on Complex Function Theory (1996), 197-201, Israel Math. Conf. Proc. 11, Bar-Ilan Univ., Ramat Gan, 1997.

[10] R. Schoen R, S. T. Yau, Lectures on Differential Geometry, Cambridge, 2004, MA: International Press.

[11] G. Szegö, Inequalities for certain eigenvalues of a membrane of given area, J. Rational Mech. Anal. 3 (1954) 343-356.

[12] H. F. Weinberger, An isoperimetric inequality for the $n$-dimensional free membrane problem, J. Rational Mech. Anal. 5 (1956) 633-636. 
[13] C. Xia, A universal bound for the low eigenvalues of Neumann Laplacians on compact domains in a Hadamard manifold. Monatsh. Math. 128 (1999), 165-171.

[14] Y. Xu, The first nonzero eigenvalue of neumann problem on Riemannian manifolds, J. Geom. Anal. 5 (1995), 151-165. 